\title{
April updates: people and places
}

\section{FELASA 2019 Award}

Werner Nicklas of the German Cancer Research Center has been chosen as the recipient of the 2019 FELASA Award from the Federation of European Animal Science Associations, in recognition of his "outstanding and achievements and extraordinary dedication... with the field of Laboratory Animal Science and for the animals used in research."

Nicklas will give the FELASA Award Lecture at the 2019 Congress, the organization's $40^{\text {th }}$, to be held in Prague, Czechoslovakia in June.

\section{Mouse microbiome grants}

The Mouse Metabolic Phenotyping Centers (MMPC) has awarded grants to six researchers interested in studying the role of the microbiome in different diseases with mouse models. The awardees and their research topics are: Joshua Stern from Albert Einstein College of Medicine, to develop a humanized mouse model to study the gut microbiome's association with urinary stone disease; Yu-Jui Yvonne Wan from University of California Davis, to study the mouse microbiome and livor tumorigenesis; Karthikeyani Chellappa from the University of Pennsylvania, to study the role of the microbiome in the metabolic benefits of NAD precursors; Susan Erdman from Massachusetts Institute of Technology, to study cholera toxin, the microbiome, and obesity; Kaikobad Irani from the University of Iowa, to study gut microbiome-regulated metabolism through MicroRNA-204; and Kalpana Acharya of Wellesley College, to study estradiol and the gut microbiome's effects on energy metabolism in female mice.

\section{NA3RsC election results} The North American 3Rs Collaborative (NA3RsC), a non-profit group of representatives from academia and industry founded to advance the 3Rs in animal research, has announced the results of its 2019 elections. Elected to leadership were: Lisa Stanislawczyk (Bristol-Myers Squibb) as Vice President; Benjamin Cappiello (AxoSim) as Vice President Elect; and Jennifer McMillan (Yerkes National Primate Research Center, Emory University) as Treasurer.

Additionally, five new members have been added to the Board of Directors: Sally Thompson-Iritani (Washington National Primate Research Center); Laura Schaevitz (Vium); Sharon Ostergaard (Bristol-Myers Squibb); Judy MacArthur-Clark (JMC Consultancy and JMC Welfare International) and Suzanne Fitzpatrick (US Food and Drug Administration).

\section{NC3Rs Fellowships announced} The NC3Rs in the UK has announced new training fellowships totaling $£ 460 \mathrm{k}$ to support four postdoctoral researchers and their studies related to the 3Rs. Diogo Mosqueira of the University of Nottingham will use CRISPR and human pluripotent stem cell-derived cardiomyocytes to study hypertrophic cardiomyopathy. Deborah Caswell of the Francis Crick Institute will focus on refining a mouse model of lung cancer through environmental enrichment. Alexandra Iordachescu of the University of Birmingham will develop a 3D, in vitro model to study bone loss. And Liane Hobson of MRC Harwell will study rodent ultrasonic vocalizations as a means to monitor and improve mouse welfare.

\section{Principle pathologist at Sinclair}

Veterinarian Alric Lopez has been appointed to the newly created role of principle anatomic veterinary pathologist at Sinclair Research, a preclinical contract research organization and provider of animal models and services based in Missouri.
He will oversee and grow the company's pathology, necropsy, and histology services.

Lopez received his board certification in pathology from the America College of Veterinary Pathologists in 2003. Prior to joining Sinclar, he has provided pathology services for Tox Path, LLC, HistoTox Labs, Envigo, and Covance Laboratories.

\section{Updates upstate}

Rensselaer, NY-based Taconic Biosciences is changing hands. The animal model and services provider has announced the appointment of a new chief executive officer. On January 14, Nancy Sandy succeeded Robert Rosenthal, now chairmen of the company's board of directors and Chief Innovation Officer. Sandy has been with Taconic since January 2016, holding prior positions at the company as senior vice president of product strategy and marketing, chief operating officer, and president.

Additionally, H.I.G. Capital, a private equity investment firm, completed its acquisition of Taconic in early February. “Taconic's scientific expertise, customer-first mentality, outstanding quality and service standards, and full suite of capabilities provide a differentiated end-to-end solution that offers unique value to the growing life sciences market," commented John Von Bargen, a Managing Director at H.I.G, in a press release. "We are excited to support Taconic's exceptional leadership team as they look to expand the Company's geographic footprint, partnership base, and product and service offerings." Sandy also commented on the acquisition by H.I.G., "H.I.G.'s investment and deep resources, particularly around M\&A, will help catalyze our continued expansion and allow us to more quickly address unmet customer needs."

Published online: 20 March 2019 https://doi.org/10.1038/s41684-019-0269-y 